\title{
Entropy Analysis in MHD Forced Convective Flow through a Circular Channel Filled with Porous Medium in the Presence of Thermal Radiation
}

\author{
Pooja Sharma ${ }^{1 *}$, Navin Kumar ${ }^{2}$ and Tarun Sharma ${ }^{3}$ \\ ${ }^{* 1}$ Department of Computer Science \& Engineering, Manipal University, Jaipur, India \\ ${ }^{2}$ Principal, Indian Military Academy, Dehradun, India \\ ${ }^{3}$ Department of Mathematics \& Statistics, Manipal University, Jaipur, India
}

Email:pooja_2383@yahoo.co.in

\begin{abstract}
The aim of the present study is to analyze the entropy generation on forced convective flow of viscous incompressible fluid flow through a circular channel filled with a hyper porous medium saturated with a rarefied gas in the presence of transverse magnetic field, thermal radiation and uniform heat flux at the walls of the channel. For the Darcian model, the velocity and heat equations are analytically solved and the effects of various physical parameters on thses are discussed. In addition, the Entropy generation rate, Nusselt number and Bejan number are analyzed for different parameters. It was perceived that entropy generation number attains high values due to the thermal radiation. This is also concluded that rate of heat transfer upsurges due to the radiative heat tansfer and Brinkmann number.
\end{abstract}

Keywords: Forced convection, Hyper porous medium, MHD, Radiation, Slip flow regime.

\section{INTRODUCTION}

Entropy is the basic concept of physics and information sciences. It is a measure to compare different state of an isolated system. Entropy is the thermodynamic variable that serves to define and relate the thermal properties of the matter and equilibrium state, basically known as second law of thermodynamics. According to the second law of thermodynamics, all real processes are irreversible. Entropy generation is a measure of the amount of irreversibility, which associated with the real processes. As entropy generation takes place, the quality of energy decreases [Cengel et al.[1]]. Bejan [2] introduced that the continuous entropy generation in the fluid flow system is occurred due to the exchange of the heat and momentum within the fluid and at the impermeable boundaries. Bejan [3], [4] discussed that entropy generation for forced convective in viscous fluid flow through the channel is due to heat transfer and viscous friction in the fluid. Entropy generation mostly occurs in the thermal and heat engineering system, therefore in the engineering field it has wide range of applications, such as industrial heat exchanger, nuclear reactor, petroleum equipment's and many other applications in the mechanical and chemical engineering. Hence lot of research carried out in last two and three decades in this field. The effect of hall current and magnetic field in a oscillatory channel flow through a rotating system was depicted recently by Ahmed and Das [5]. Entropy generation in a laminar fluid flow through a circular pipe is investigated by Ahmet et al. [6]. Yurusoy et al. [7] studied the effects of entropy generation and temperature dependent viscosity on a nonNewtonian fluid flow in annular pipes. Entropy generation for forced convection in a porous saturated circular tube with uniform wall temperature was discussed by Hooman et al. [8]. Pakdemirli et al. [9] analyzed the entropy generation in pipe due to non-Newtonian fluid flow in the case of constant viscosity. They concluded that an increment in the nonNewtonian parameters, reduces the fluid friction in the region close to the pipe wall. Natural convection and entropy generation of nano-fluids in a square cavity was concluded by Bouchoucha and Bessaih [10]. Unsteady heat and mass transfer effects on an impulsively started infinite vertical plate in the presence of porous medium was studied by Loganathan et al. [11]. Ganesan et al. [12] considered the Radiation and mass transfer effects on flow of an incompressible viscous fluid past a moving vertical cylinder. Their work was extended by Suneeta et al. [13] for MHD fluid flow. On the same fluid flow conditions, the entropy generation was analyzed by Mahian et al. [14]. Tshehla et al. [15] premeditated the entropy generation in a variable viscosity fluid flow between two concentric pipes with a convective cooling at the surface. Aldos et al. [16] discussed the mixed convection from a horizontal cylinder in a porous medium. Effect of slip conditions on forced convection and entropy generation in a circular channel occupied by a highly porous medium Darcy extended Brinkmann Forchheimer model was conferred by Chauhan et al. [17]. Kumar Navin et al. [18] extended this work. They concluded the MHD forced convection and entropy generation in a circular channel occupied by hyper porous medium. 
Motivated by the above cited research work, we have made an attempt to analyze the entropy generation on forced convective flow of viscous incompressible electrically conducting fluid through a circular channel filled with a hyper porous medium saturated with a rarefied gas in the presence of transverse magnetic field, thermal radiation and uniform heat flux at the walls of the channel.

\section{FORMULATION OF THE PROBLEM}

Consider a steady two dimensional flow of a viscous incompressible electrically conducting fluid along the $\bar{x}$ direction taken as the axis of circular channel. A uniform magnetic field is applied in the transverse direction of the channel.

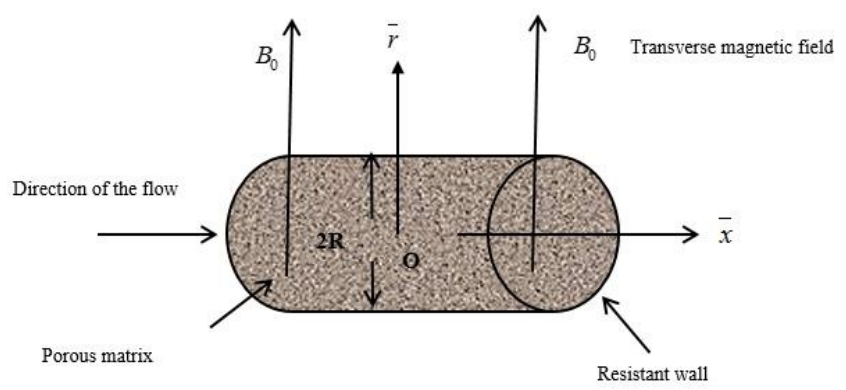

Figure 1. Geometry configuration

The internal territory of the circular channel with radius $R$ is employed with extremely absorbent medium soaked with rarefied gas. The wall of the circular channel is supposed as impermeable or resistant wall. It is also assumed that the radiation heat flux in the $\bar{x}$ direction is negligible as compared to the direction perpendicular to the fluid flow. By using the Darcy-extended Brinkmann Forchheimer model of porous medium for steady MHD forced convective flow through the circular channel in the presence of thermal radiation the governing equations of the flow are given as below:

$\mu_{e f f}\left(\frac{d^{2} \bar{u}}{d \bar{r}^{-2}}+\frac{1}{\bar{r}} \frac{d \bar{u}}{d \bar{r}}\right)-\frac{\mu}{\bar{K}} \bar{u}-\sigma B_{0}^{2} \bar{u}-\frac{C_{i} \rho \bar{u}^{2}}{\sqrt{\bar{K}}}+\mathrm{P}=0$,

$\rho C_{p} \bar{u} \frac{d \bar{T}}{d \bar{x}}=\frac{\kappa}{\bar{r}} \frac{\partial}{\partial \bar{r}}\left(\bar{r} \frac{d \bar{T}}{d \bar{r}}\right)+\frac{\mu}{\bar{K}} \bar{u}^{2}+\mu_{e f f}\left(\frac{d \bar{u}}{d \bar{r}}\right)^{2}+$

$+\sigma B_{0}^{2} \bar{u}^{2}-\frac{1}{\bar{r}} \frac{\partial}{\partial \bar{r}}\left(\bar{r} q_{r}\right)$,

The corresponding boundary/symmetry conditions are

$\bar{r}=0: \frac{d \bar{u}}{d \bar{r}}=0, \frac{d \bar{T}}{d \bar{r}}=0$,
$\bar{r}=R: \bar{u}=-\bar{\alpha} \frac{d \bar{u}}{d \bar{r}}, \bar{T}=-\bar{\beta} \frac{d \bar{T}}{d \bar{r}}$

Following the Rosseland approximation Brewster [19] which leads to the radiative heat flux $q_{r}$ is given by $q_{r}=-\frac{4 \sigma}{3 \phi} \frac{\partial \bar{T}^{4}}{\partial r}$

If temperature difference within the flow is sufficiently small such that $\bar{T}^{4}$ may be expressed as a linear function of the temperature, then the Taylor series for $\bar{T}^{4}$ about $\bar{T}_{w}$ after neglecting the higher order terms, is given as

$\bar{T}^{4} \cong 4 \bar{T}_{w}^{4} \bar{T}-3 \bar{T}_{w}^{4}$.

In view of equations (4) and (5), equation (2) is reduced as

$\rho C_{p} \bar{u}\left(\frac{\partial \bar{T}}{\partial \bar{x}}\right)=\left(\kappa+\frac{16 \sigma \bar{T}_{w}^{3}}{3 \phi}\right) \cdot\left\{\frac{\partial^{2} \bar{T}}{\partial \bar{r}^{2}}+\frac{1}{\bar{r}} \frac{\partial \bar{T}}{\partial \bar{r}}\right\}+$

$+\frac{\mu}{\bar{K}} \bar{u}^{2}+\mu_{e f f}\left(\frac{\partial \bar{u}}{\partial \bar{r}}\right)^{2}+\sigma B_{0}^{2} \bar{u}^{2}$.

\section{METHOD OF SOLUTION}

We now introduce the following dimensionless quantities as

$$
\begin{aligned}
& z=\frac{\bar{z}}{P e R}, r=\frac{\bar{r}}{R}, u=\frac{\mu \bar{u}}{\mathrm{PR}^{2}}, \mu_{1}=\frac{\mu_{e f f}}{\mu}, D a=\frac{\bar{K}}{R^{2}}, \eta=\sqrt{\frac{1}{D a}}, \\
& F=\frac{\rho C_{i} \mathrm{P} R^{3}}{\mu^{2}}, P e=\frac{\rho C_{p} R \bar{U}_{\text {mean }}}{\kappa}, \alpha=\frac{\bar{\alpha}}{R}, \beta=\frac{\bar{\beta}}{R}, M=\sqrt{\frac{\sigma B_{0}^{2} R^{2}}{\mu}} .
\end{aligned}
$$

Using (7), the equation (1) is reduced in dimensionless form

$\frac{d^{2} u}{d r^{2}}+\frac{1}{r} \frac{d u}{d r}-\frac{\eta^{2}}{M} u-\frac{M^{2}}{\mu_{1}} u-\frac{F \eta}{\mu_{1}}+\frac{1}{\mu_{1}}=0$,

The corresponding boundary conditions for velocity field became as

at $r=0: \frac{d u}{d r}=0, r=1: u=-\alpha \frac{d u}{d r}$.

Further defining the mean velocity $\bar{U}_{\text {mean }}$ and bulk mean temperature $\bar{T}_{\text {mean }}[$ Navin et al. $[18]]$.

$\bar{U}_{\text {mean }}=\frac{2}{R^{2}} \int_{0}^{R} \bar{u} \bar{r} d \bar{r}$ and $\bar{T}_{\text {mean }}=\frac{2}{R^{2} \bar{U}} \int_{0}^{R} \bar{u} \bar{T} \bar{r} d \bar{r}$.

The dimensionless variables are originated as

$u=\frac{\bar{u}}{\bar{U}_{\text {mean }}}, T=\frac{\bar{T}-\bar{T}_{w}}{\bar{T}_{\text {mean }}-\bar{T}_{w}}, N u=\frac{2 R q_{w}}{\kappa\left(\bar{T}_{w}-\bar{T}_{\text {mean }}\right)}$,

$T$ is the function of radial coordinate $(\bar{r})$ only, while the bulk mean temperature $\bar{T}_{\text {mean }}$ is the function of axial 
coordinate $(\bar{x})$ only. Now from the first law of thermodynamics, for uniform heat flux on the wall, we have

$\frac{\partial \bar{T}}{\partial \bar{x}}=\frac{2 q_{w}}{\rho C_{p} R \bar{U}_{\text {mean }}}$

Now using equation (11) and (12), equation (6) is reduced as

$\frac{d^{2} T}{d r^{2}}+\frac{1}{r} \frac{d T}{d r}+(N u) u L+\eta^{2} B r u^{2} L+B r \mu_{R} L\left(\frac{d u}{d r}\right)^{2}+$

$+M^{2} \mathrm{Bru}^{2} L=0$,

where $B r=\frac{\mu \bar{U}_{\text {mean }}^{2}}{\kappa\left(\bar{T}_{\text {mean }}-\bar{T}_{w}\right)}$ is the Brinkmann number, $N=\frac{\kappa \phi}{4 \sigma \bar{T}_{w}^{3}}$ the Radiation parameter [Ganeshan et.al [12]] and $L=\frac{3 N}{3 N+4}$. The corresponding boundary/symmetric conditions for temperature field are:

$$
r=0: \frac{d T}{d r}=0, \quad r=1: T=-\beta \frac{d T}{d r}
$$

The Nusselt number can be found by substituting $u$ and $T$ in the compatibility condition

$\int_{0}^{1} u T r d r=\frac{1}{2}$

This is an identity from the first law of thermodynamics [Nield et al. [20]].

\subsection{Solution of momentum equation}

In case of large Darcy number, we write the following asymptotic expression for the velocity distribution with the assumption $\eta<<1$

$u(r)=u_{0}(r)+\eta u_{1}(r)+o\left(\eta^{2}\right)$.

Now substituting the equation (16) into (8) and equating the coefficient of like powers of $\eta$, we find the following set of coupled differential equations:

$\frac{d^{2} u_{0}}{d r^{2}}+\frac{1}{r} \frac{d u_{0}}{d r}-\frac{M^{2}}{\mu_{1}} u_{0}+\frac{1}{\mu_{1}}=0$,

$\frac{d^{2} u_{1}}{d r^{2}}+\frac{1}{r} \frac{d u_{1}}{d r}+\frac{M^{2}}{\mu_{1}} u_{1}-\frac{F}{\mu_{1}} u_{0}^{2}=0$,

and the corresponding boundary conditions are

$r=0: \frac{d u_{0}}{d r}=0, \quad \frac{d u_{1}}{d r}=0$, $r=1: u_{0}=-\alpha \frac{d u_{0}}{d r}, u_{1}=-\alpha \frac{d u_{1}}{d r}$

For the diminutive magnetic field, $u_{0}$ and $u_{1}$ can be expanded as

$u_{0}(r)=u_{00}(r)+M^{2} u_{01}(r)+o\left(M^{4}\right)$,

$u_{1}(r)=u_{10}(r)+M^{2} u_{11}(r)+o\left(M^{4}\right)$.

Substituting the equation (20) in equation (17) and (18) and equating the coefficient of like power of $M^{2}$ and neglecting the higher order of $M^{2}$, we have

$\frac{d^{2} u_{00}}{d r^{2}}+\frac{1}{r} \frac{d u_{00}}{d r}+\frac{1}{\mu_{1}}=0$

$\frac{d^{2} u_{01}}{d r^{2}}+\frac{1}{r} \frac{d u_{01}}{d r}-\frac{u_{00}}{\mu_{1}}=0$

$\frac{d^{2} u_{10}}{d r^{2}}+\frac{1}{r} \frac{d u_{10}}{d r}-\frac{F}{\mu_{1}} u_{00}^{2}=0$,

$\frac{d^{2} u_{11}}{d r^{2}}+\frac{1}{r} \frac{d u_{11}}{d r}=\frac{u_{10}}{\mu_{1}}=\frac{2 F}{\mu_{1}} u_{00} u_{01}$.

The corresponding boundary conditions are given by

$r=0: \frac{d u_{00}}{d r}=0, \frac{d u_{01}}{d r}=0, \frac{d u_{10}}{d r}=0, \frac{d u_{11}}{d r}=0 ;$

$r=1: u_{00}=-\alpha \frac{d u_{00}}{d r}, u_{01}=-\alpha \frac{d u_{01}}{d r}$,

$u_{10}=-\alpha \frac{d u_{10}}{d r}, u_{11}=-\alpha \frac{d u_{11}}{d r}$.

Equations (21) to (24) are coupled linear differential equations with variable coefficient. These are solvable by straight forward calculation and the solution is given by

$u(r)=A_{23}+r^{2} A_{24}+r^{4} A_{25}+r^{6} A_{26}+r^{8} A_{27}$.

where $A_{23}$ to $A_{27}$ are constants.

\subsection{Solution of heat equation}

Now by using equation (26) in the mean velocity equation (10), we get

$\bar{U}_{\text {mean }}=\frac{\mathrm{P} R^{2}}{60 \mu}\left(A_{28}+\eta A_{29}\right)$

where $A_{23}$ to $A_{29}$ are constants and not given here due to sake of bravity. Similarly, for the large Darcy number, we write the following asymptotic expression for temperature distribution as

$T(r)=T_{0}(r)+\eta T_{1}(r)+o\left(\eta^{2}\right)$. 
Using (27), (28) and (10) in equation (13), leaving the higher order than two, we obtained the following set of differential equations

$$
\begin{aligned}
& \frac{d^{2} T_{0}}{d r^{2}}+\frac{1}{r} \frac{d T_{0}}{d r}+\frac{60 N u u_{0}}{A_{28}} L+\frac{3600 B r \mu_{1}}{A_{28}^{2}}\left(\frac{d u_{0}}{d r}\right)^{2} L+ \\
& +\frac{3600 B r M^{2} u_{0}^{2}}{A_{28}^{2}} L=0,
\end{aligned}
$$

$\frac{d^{2} T_{1}}{d r^{2}}+\frac{1}{r} \frac{d T_{1}}{d r}+\frac{60 N u}{A_{28}}\left\{u_{1}-\frac{A_{28}}{A_{29}} u_{0}\right\} L+$

$+\frac{7200 B r \mu_{1}}{A_{28}^{2}}\left[\frac{d u_{0}}{d r}\left\{\frac{d u_{1}}{d r}-\frac{A_{29}}{A_{28}}\left(\frac{d u_{0}}{d r}\right)\right\}\right] L+$

$+\frac{7200 B r M^{2} u_{0}}{A_{28}}\left\{u_{1}-\frac{A_{29}}{A_{28}} u_{0}\right\} L=0$.

The corresponding boundary conditions are:

at $r=0: \frac{d T_{0}}{d r}=0, \frac{d T_{1}}{d r}=0$

at $r=1: T_{0}=-\beta \frac{d T_{0}}{d r}, T_{1}=-\beta \frac{d T_{1}}{d r}$.

Equation (29) and (30) are ordinary linear differential equations with variable coefficient these are solvable by usual algebric method.

Finally, solving the equation (15) for determining the Nusselt number, we find

$$
N u=\frac{A_{28}+\eta A_{29}}{120 A_{106}}-\frac{A_{105}}{A_{106}} .
$$

where $A_{30}$ to $A_{106}$ be constants.

\section{ENTROPY GENERATION}

Entropy generation analysis concerned with the MHD forced convective flow through a circular channel filled with porous medium in the presence of thermal radiation is considered. The volumetric rate of entropy generation is obtained based on the previous discussed studies of the fluid flow problems for velocity and temperature field given by Woods [21].

$E_{G}=\left(E_{G}\right)_{H}+\left(E_{G}\right)_{F}+\left(E_{G}\right)_{M}$.

where $\left(E_{G}\right)_{H},\left(E_{G}\right)_{F}$ and $\left(E_{G}\right)_{M}$ represent the entropy generation rate due to heat transfer, fluid friction and magnetic field respectively, and their values for the present problem in cylindrical coordinates are given by

$\left(E_{G}\right)_{H}=\frac{\kappa}{\bar{T}_{0}^{2}}\left[\left(\frac{\partial \bar{T}}{\partial \bar{r}}\right)^{2}+\left(\frac{\partial \bar{T}}{\partial \bar{x}}\right)^{2}\right]$,
$\left(E_{G}\right)_{F}=\frac{1}{\bar{T}_{0}}\left[\frac{\mu \bar{u}^{2}}{\bar{K}}+\mu_{e f f}\left(\frac{d \bar{u}}{d \bar{r}}\right)^{2}\right]$,

$\left(E_{G}\right)_{M}=\frac{\sigma B_{0}^{2} \bar{u}^{2}}{\bar{T}_{0}}$.

where $\bar{T}_{0}$ is the reference temperature.

Now using the non-dimensional quantities defined in equation (7) and (11), we get entropy generation in dimensionless form as:

$$
\begin{aligned}
& N s=\frac{E_{G}}{E_{0}}=\left[\left(\frac{d T}{d r}\right)^{2}+\left(\frac{N u}{P e}\right)^{2}\right]+ \\
& +T_{0} B r\left[\frac{u^{2}}{D a}+\mu_{R}\left(\frac{d u}{d r}\right)^{2}\right]+T_{0} B r M^{2} u^{2} .
\end{aligned}
$$

where $\theta_{0}=\frac{\bar{T}_{0}}{\left(\bar{T}_{\text {mean }}-\bar{T}_{w}\right)}$ is the dimensional reference temperature and $E_{0}=\frac{\kappa}{\theta_{0}^{2} R^{2}}$ the reference volumetric entropy generation. Equation (37) can be written as

$N s=N s_{1}+N s_{2}+N s_{3}$,

where entropy generation due to heat transfer

$N s_{1}=\left[\left(\frac{d T}{d r}\right)^{2}+\left(\frac{N u}{P e}\right)^{2}\right]$,

entropy generation due to viscous dissipation

$N s_{2}=T_{0} B r\left[\frac{u^{2}}{D a}+\mu_{R}\left(\frac{d u}{d r}\right)^{2}\right]$,

and entropy generation due to magnetic field

$N s_{3}=T_{0} B r M^{2} u^{2}$

The Bejan number $(B e)$ is given by

$B e=\frac{N s_{1}}{N s}$

which compares the magnitude of entropy generation due to heat transfer to the magnitude of the total entropy generation.

\section{RESULTS AND DISCUSSION}

In the present study, an analytical solution is obtained for the velocity and temperature profiles for the fully developed steady MHD forced convective flow through a circular 
channel filled with a hyper porous medium soaked with a rarefied gas. The entropy generation due to heat transfer, fluid friction and magnetic field is evaluated numerically for different values of governing parameters.

Figure 2 shows the effect of magnetic field and velocity slip parameter on the velocity field. It is depicted that due to the intensity of the magnetic field, the fluid velocity is declining gradually. While slip parameter upsurges velocity field. This is quite visible that the fluid has its high velocity in the centre of the circular tube.

It is observed from Figure 3 that an increase in Forchheimer number $\mathrm{F}$ reduces the fluid velocity. But this demotion is comparatively large near the centre line. The dimensionless temperature profiles are plotted against $r$ in Figure from 4 to 7 . From Figure 4 it is observed that an increment in the temperature slip parameter $\beta$ supports the magnitude of the temperature while reverse effect is seen due to the enhancement in velocity slip parameter $\alpha$.

Figure 5 describes that when the Brinkmann number $\mathrm{Br}$ and intensity of magnetic field $\mathrm{M}$ increase, the fluid temperature rises gradually and this effect is more in the centre line of the channel. From Figure 6 it is predicted that with the increasing of Forchheimer number $\mathrm{F}$, the temperature of the fluid is declining. The effect of radiation parameter on temperature fluid can be seen clearly from Figure 7 . The fluid temperature is getting increased due to rise in thermal radiation parameter $\mathrm{N}$. This promotion is comparatively higher on the centre line of the circular channel.

Now Figure 8 to 12 illustrate the effect of various physical parameter on entropy generation rate. From Figure 8 we observed that the entropy generation rate is declining due to increase in the Forchheimer number. While Figure 9 to 11 predict that an increase value of temperature slip parameter $\beta$, Brinkmann number $\mathrm{Br}$ and Hartmann number $\mathrm{M}$ upsurging the entropy generation rate. Figure 12 shows that effect of radiation parameter on entropy generation rate. This is getting increased due to increase of the thermal radiation in the presence of uniform magnetic field.

Figure 13 to 16 reflect the effect of various physical parameter on Bejan number $\mathrm{Be}$. The Bejan number attains its maximum value (i.e., 1) for all value of $r$. Figure 13 illustrate that Bejan number increases due to an increment in the Brinkmann number. Figure 14 and 15 depict that an increment in the Forchheimer number and Hartmann number raise the value of Bejan number. Figure 16 shows that an increment in the thermal radiation parameter $\mathrm{N}$ support the Bejan number.

Table 1 describes that the rate of heat transfers in terms of the Nusselt number rises with an increase of temperature slip parameter $\beta$, Brinkmann number $\mathrm{Br}$, Forchheimer number $\mathrm{F}$, viscosity ratio $\mu_{1}$, Hartmann number $M$, and radiation parameter N. While it reduces with the increase value of velocity slip parameter $\alpha$.

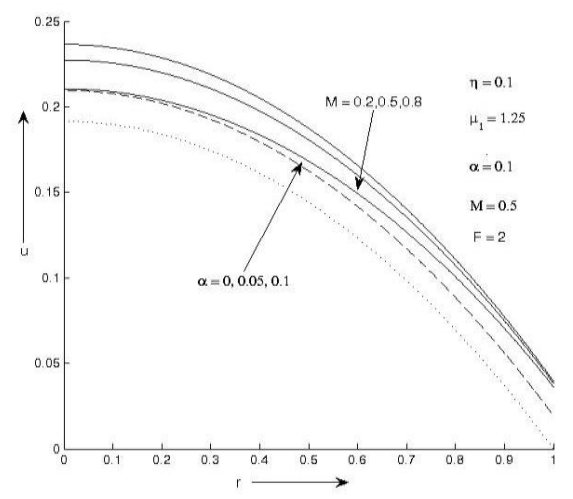

Figure 2. Velocity versus $r$

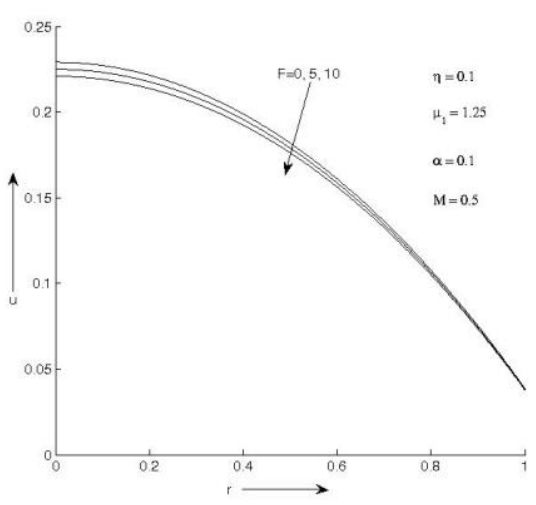

Figure 3. Velocity versus $r$

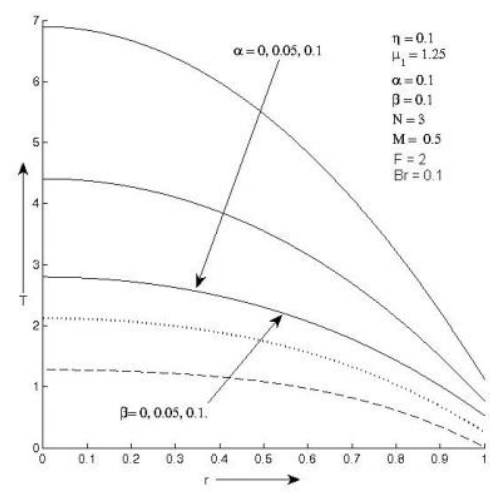

Figure 4. Temperature versus $r$

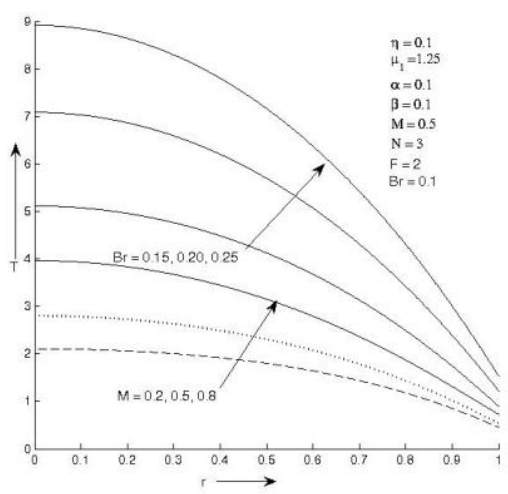

Figure 5. Temperature versus $r$ 


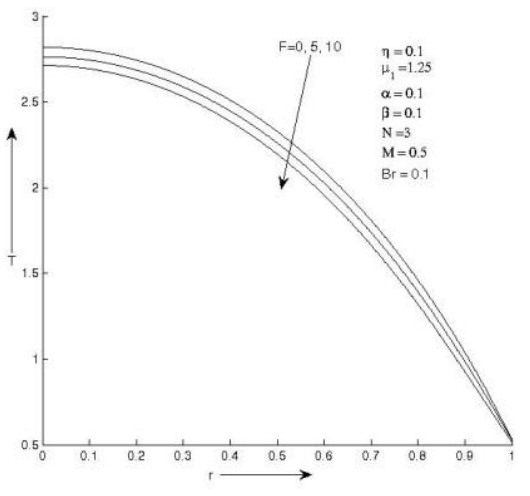

Figure 6. Temperature versus $r$

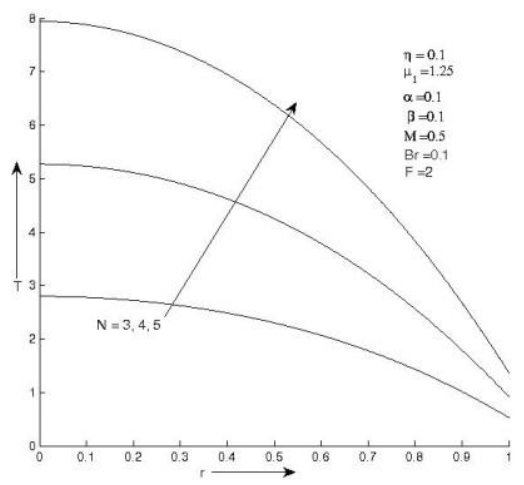

Figure 7. Temperature versus $r$

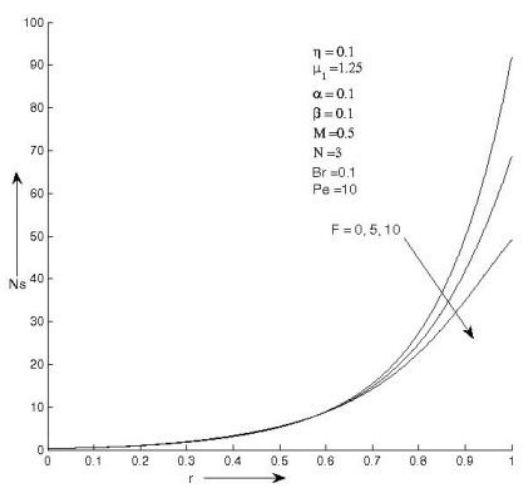

Figure 8. Ns versus $r$

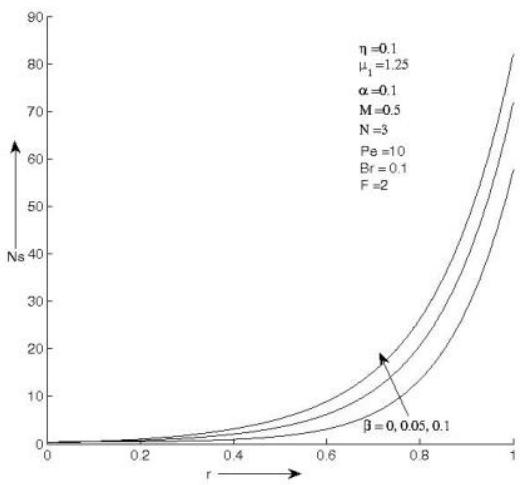

Figure 9. Ns versus $r$

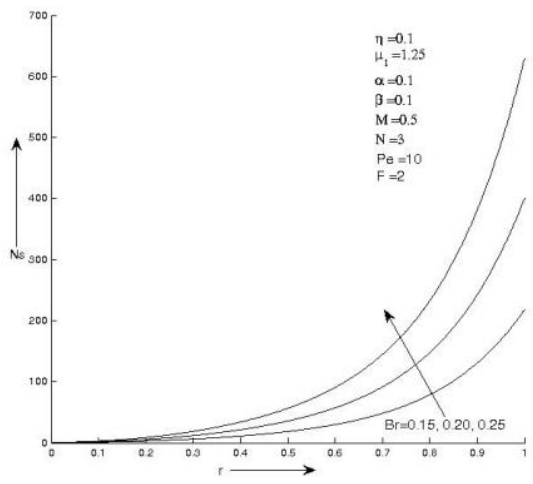

Figure 10. Ns versus $r$

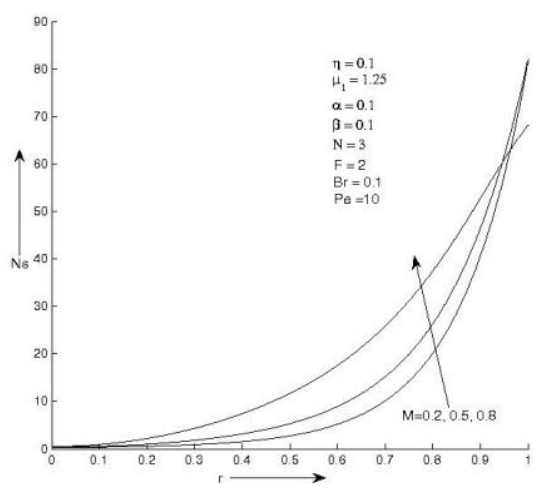

Figure 11. Ns versus $r$

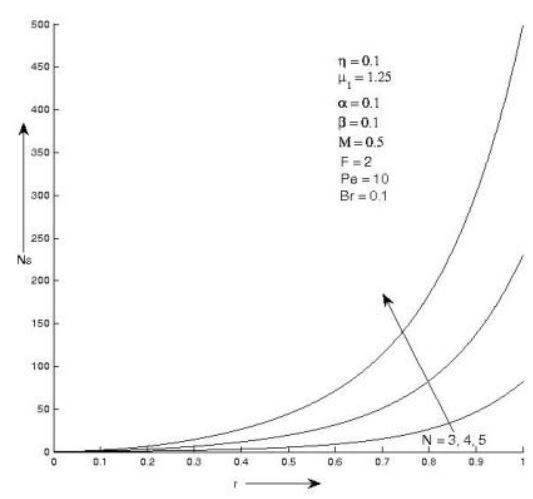

Figure 12. Ns versus $r$

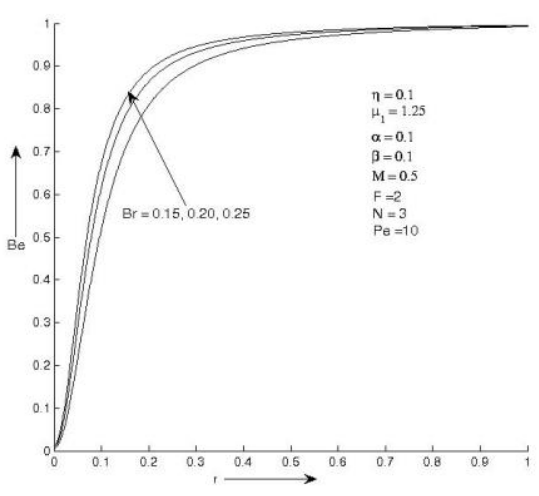

Figure 13. Be versus $r$ 


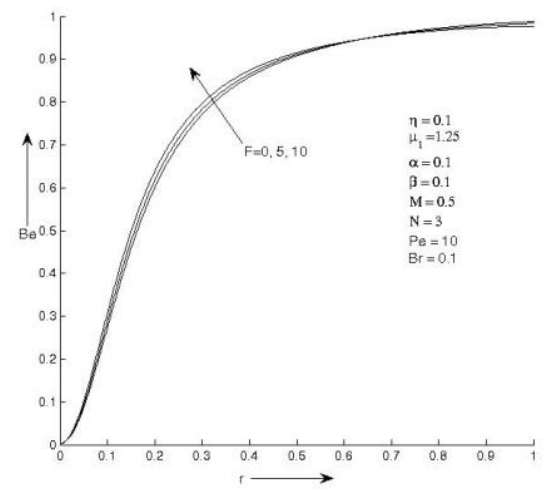

Figure 14. Be versus $r$

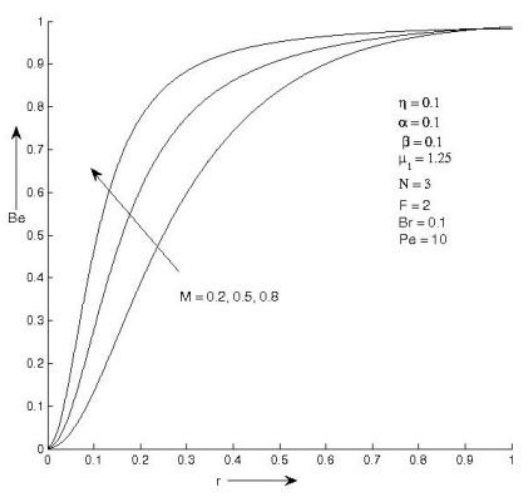

Figure 15. Be versus $r$

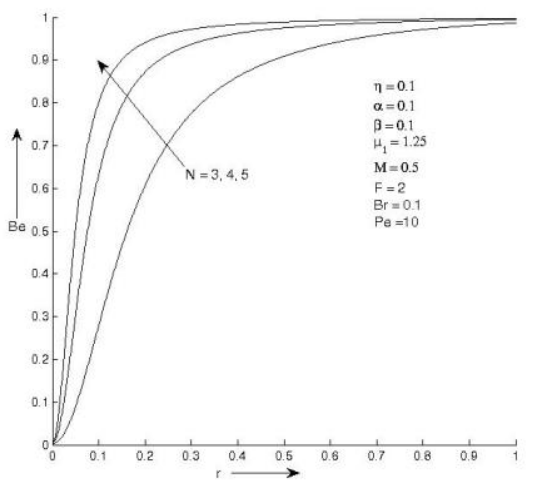

Figure 16. Be versus $r$

Table 1. Nusselt number $(\mathrm{Nu})$ at wall when $\eta=0.1$

\begin{tabular}{|l|l|l|l|l|l|l|l|}
\hline$\alpha$ & $\beta$ & $\mathrm{Br}$ & $\mathrm{F}$ & $\mu_{1}$ & $\mathrm{M}$ & $\mathrm{N}$ & $\mathrm{Nu}$ \\
\hline 0.05 & 0.05 & 0.1 & 2 & 1.25 & 0.4 & 3 & 0.4023 \\
\hline 0.1 & 0.05 & 0.1 & 2 & 1.25 & 0.4 & 3 & 0.1532 \\
\hline 0.05 & 0.1 & 0.1 & 2 & 1.25 & 0.4 & 3 & 0.4844 \\
\hline 0.05 & 0.05 & 0.2 & 2 & 1.25 & 0.4 & 3 & 1.1044 \\
\hline 0.05 & 0.05 & 0.1 & 5 & 1.25 & 0.4 & 3 & 0.4047 \\
\hline 0.05 & 0.05 & 0.1 & 2 & 2 & 0.4 & 3 & 0.8267 \\
\hline 0.05 & 0.05 & 0.1 & 2 & 1.25 & 0.5 & 3 & 0.4428 \\
\hline 0.05 & 0.05 & 0.1 & 2 & 1.25 & 0.4 & 4 & 0.5295 \\
\hline 0.05 & 0.05 & 0.1 & 2 & 1.25 & 0.4 & 5 & 0.5556 \\
\hline
\end{tabular}

\section{CONCLUSIONS}

An investigation has been carried out to analyze the entropy generation on forced convective flow of viscous incompressible fluid flow through a circular channel filled with a hyper porous medium in the presence of transverse magnetic field and thermal radiation. The study concludes that

(1) Entropy generation rate is rising due to increase in temperature slip parameter, viscosity ratio, Hartmann number and radiation parameter.

(2) Entropy generation rate is declining due to the presence of velocity slip coefficient, Brinkmann number and Forchheimer number.

(3) The value of the Bejan number is increasing due to the effect of Brinkmann number, Forchheimer number, Hartmann number and radiation parameter.

\section{REFERENCES}

[1] Y. A. Cengel and M. A Boles, Thermodynamics of Engineering Approach, New York: Mc Graw Hill, 1994.

[2] A. Bejan, Entropy Generation through Heat and Fluid Flow, New York: Willy, 1982.

[3] A. Bejan, "A study of entropy generation in fundamental convective heat transfer," ASME Journal of Heat Transfer, vol. 101, pp. 718-725, 1979. DOI: $\underline{10.1115 / 1.3451063}$.

[4] A. Bejan, Entropy Generation Minimization, New York: CRC Press, 1996.

[5] N. Ahmed and S. M. Das, "Oscillatory MHD Mass Transfer channel flown in a rotating system with hall current", International Journal of Heat and Technology, vol. 34, no.1, pp. 115-123, 2016. DOI: 10.18280/ijht.340117.

[6] Z. Ahmet and Rached Ban-Mansour, "Entropy generation in laminar fluid flow through a circular pipe," Entropy, vol. 5, pp. 404-406, 2003. DOI: 10.3390/e5050404.

[7] M. Yurusoy, B. S. Yilbas and M. Pakdemirli, "NonNewtonian fluid flow in annular pipes and entropy generation: Temperature dependent viscosity," Sadhana, vol. 31, no.6, pp. 683-695, 2006. DOI: 10.1007/BF02716888.

[8] K. Hooman and A. Ejlali, "Entropy generation for forced convection in a porous saturated circular tube with uniform wall temperature," International Journal of Communication in Heat and Mass Transfer, vol. 34, pp. 408-419, $2007 . \quad$ DOI: 10.1016/j.icheatmasstransfer.2006.10.008.

[9] M. Pakdemirli and B. S. Yilbas, "Entropy generation in pipe due to non-Newtonian fluid flow: Constant viscosity case," Sadhana, vol. 31, no. 1, pp. 21-29, 2006. DOI: $10.1007 / \mathrm{BF} 02703797$.

[10] A. M. Bouchoucha and R. Bessaih, "Natural convection and entropy generation of nano-fluids in a square cavity," International Journal of Heat and Technology, vol. 33, no. 4, pp. 1-10, 2015. DOI: 10.18280/ijht.330401.

[11] P. Loganathan and C. Sivapoornapriya, "Unsteady heat and mass transfer effects on an impulsively started infinite vertical plate in the presence of porous medium," International Journal of Heat and 
Technology, vol. 33, no. 2, pp. 69-74, 2015. DOI: 10.18280/ijht.330211.

[12] P. Ganesan and P. Loganathan, "Radiation and mass transfer effects on flow of an incompressible viscous fluid past a moving vertical cylinder," International Journal of Heat and Mass Transfer, vol. 45, pp. 42814288, 2002. DOI: 10.1016/S00179310(02)00114-0.

[13] S. Suneeta and N. Reddy Bhasker, "Radiation and mass transfer effect on MHD free convective flow past a moving vertical cylinder in a porous medium," Journal of Naval Architerature and Marin Engineering, pp. 1-10, 2010. DOI: 10.3329/jname. v7i1.2901.

[14] O. Mahian, H. Oztop, L. Pop, S. Mahmud and S. Wongwises, "Entropy generation between two vertical cylinder in the presence of MHD flow subjected to constant wall temperature," International Communication of Heat and Mass Transfer, vol. 44, pp. 87-92, 2013. DOI: 10.1016/j.icheatmasstransfer.2013.03.005.

[15] M. S. Tshehla and O. D. Makinde, "Analysis of entropy generation in a variable viscosity fluid flow between two concentric pipes with a convective cooling at the surface," International Journal of Physical Sciences, vol. 6, no. 25, pp. 6053-6060, 2011. DOI: $10.5897 / \mathrm{IJPS} 11.889$.

[16] T. K. Aldos and Y. D. Ali, "MHD mixed convection from a horizontal cylinder in a porous medium," JSME International Journal Series, vol. 40, no. 2, pp. 290295, 1997. DOI: 10.1299/jsmeb.40.290.

[17] D. S. Chauhan and V. Kumar, "Effect of slip conditions on forced convection and entropy generation in a circular channel occupied by a highly porous medium Darcy extended BrinkmannForchheimer model," Turkish Journal Engineering Environment Sciences, vol. 33, pp. 91-104, 2009. DOI: 10.3906/muh-0903-9.

[18] N. Kumar and S. Gupta, "MHD forced convection and entropy generation in a circular channel occupied by hyper porous medium," Heat and Technology, vol. 29, no. 1, pp. 91-100, 2011.

[19] M. Q. Brewster, Thermal Radiative Transfer and Properties, New York: John Willy and Sons, 1992.

[20] D. A. Nield and A. V. Kuznetsov, "Effect of heterogeneity in forced convection in a porous medium: Parallel plate channel or circular duct," International Journal of Heat and Mass Transfer, vol. 43, pp. 4119-4134, 2000. DOI: 10.1016/S00179310(00)00025-9.

[21] L. C. Woods, Thermodynamics of Fluid Systems, Oxford: Oxford University Press, 1975.

\section{NOMENCLATURE}

$\begin{array}{ll}B r & \text { brinkmann number } \\ B e & \text { bejan number } \\ C_{p} & \text { specific heat, J. kg-1. K-1 } \\ D a & \text { darcy number } \\ F & \text { forchheimer number } \\ \kappa & \text { thermal conductivity, W.m-1. K-1 } \\ \bar{K} & \text { permeability } \\ M & \text { hartman number } \\ N & \text { radiation parameter } \\ N s & \text { entropy generation coefficient } \\ N u & \text { nusselt number } \\ P & \text { negative of applied pressure gradient in } \\ & \bar{x} \text { direction } \\ P e & \text { peclet number } \\ q_{r} & \text { rediative heat flux } \\ R & \text { radius of circular channel } \\ \bar{r} & \text { radial coordinate of cylinder } \\ T & \text { dimensionless temperature } \\ \bar{T}_{\text {mean }} & \text { bulk mean temperature } \\ T_{w} & \text { temperature at wall } \\ \bar{u} & \text { fluid velocity } \\ \bar{U}_{\text {mean }} & \text { mean velocity } \\ & \end{array}$

\section{Greek symbols}

$\begin{array}{ll}\frac{\alpha}{\alpha} & \begin{array}{l}\text { dimensionless velocity slip coefficient } \\ \text { velocity slip coefficient }\end{array} \\ \bar{\beta} & \text { dimensioless temperature slip coefficient } \\ \mu & \text { temperature slip coefficient } \\ \mu_{\text {eff }} & \text { dynamic viscosity, kg. m-1.s-1 } \\ \mu_{1} & \text { effective viscosity } \\ \sigma & \text { viscosity ratio } \\ \rho & \text { coefficient of electrical consuctivity } \\ \eta & \text { Fluid density } \\ \phi & \text { porous media shape parameter }\end{array}$

\section{Subscripts}

$\begin{array}{ll}\mathrm{w} & \text { wall } \\ p & \text { pressure } \\ \text { eff } & \text { effective }\end{array}$

